\title{
Mass determination of astrometric binaries with Hipparcos ${ }^{\star}$
}

\section{Theory and simulation}

\author{
C. Martin, F. Mignard, and M. Froschlé \\ Observatoire de la Côte d'Azur, Dept. CERGA, URA CNRS 1360, Av. N. Copernic, F-06130 Grasse, France
}

Received June 4; accepted August 20, 1996

\begin{abstract}
The analysis of the observations of double stars made by the ESA satellite Hipparcos has involved a very specific processing to derive the relevant astrometric parameters. This required to distinguish between several categories of double stars according to the separation and orbital motion. We show that for close pairs with orbital period less than about 20 years, the concept of photocentric orbit of an astrometric binary needs to be generalized to benefit fully from the accuracy of Hipparcos. We introduce a point more naturally associated with the Hipparcos observations, the hippacentre, whose orbital path is not longer similar to the relative keplerian orbit of the components, unlike that of the photocentre. For systems with separation larger than about $00^{\prime \prime} 3$, it is possible to determine separately the mass and the intensity ratio of the components from the absolute path of the hippacentre on the sky. For smaller separations the scale of the photocentric orbit is recovered as a limiting case. The scope of this paper is to set forth the principles of the method and to explore its possibilities and limitations from extensive simulations.
\end{abstract}

Key words: methods: data analysis - binaries: visual — stars: fundamental parameters

\section{Introduction}

The reduction of the observations carried out by the Hipparcos satellite came recently to an end and the availability of the final results to the scientific community is due in early 1997. The merging of the two solutions produced independently by the Consortiums FAST and NDAC (Kovalevsky et al. 1992, 1995; Lindegren et al.

Send offprint requests to: C. Martin

* Based on observations made by the ESA Hipparcos satellite.
1992) provides a single set of astrometric and photometric estimates for each program star, including the analysis of their variability and, of more relevance in the present context, an astrometric solution of the double and multiple stars. These stars were either known as double before the mission, thanks to an intense data compilation of existing information (Dommanget 1985, 1989) or discovered during the analysis of the Hipparcos observations.

It is currently believed that half of all stars are binary, sharing a common proper motion, or put differently that more stars are to be found in association than in isolation. This is a very loose concept since within this idea we cannot rule out that the Sun belongs to such a pair. If we restrict to pairs effectively seen in gravitational interaction from the existence of a non rectilinear absolute motion, the number of true binary objects decreases considerably, may be as most orbital periods are so long that the curvilinear motion has gone unnoticed so far. It is customary to divide the binary stars into broad classes defined by the observational means by which they were recognized, such as visual, eclipsing, spectroscopic or astrometric binaries, rather than by any more physical criterion pertaining to the structure of the couple.

In the case of Hipparcos there were basically two ways to recognize that a star has a nearby companion:

- First when the photometric signal of a program star measured on the grid departed significantly from the well calibrated single star signal. Thus statistical tests could be constructed to determine at an early level of the reduction process whether a particular object had to be removed from the single stars basket. This recognition made no use of the absolute motion of the components or, of the photocentre, against the stellar background. It relied only on the recognition that the entry which was being observed was either an extended source or composed of several star-like sources. In this respect this procedure of detection was very akin to the telescopic detection of a visual binary by simple 
inspection, although in the Hipparcos case this was done by an image processing rather than by the eye. In the following these stars will be referred to as resolved double stars. About 12000 such systems have been solved from the Hipparcos observations and an additional 6000 are flagged as suspected non singles.

- Alternatively, when the image could not be distinguished from that of a point like source, even though the source might have been a close pair, the combined signal was analyzed in the same way as that of a single star, with the basic assumption that the absolute motion was rectilinear, (see Wielen 1996, for exceptions to this rule). However this assumption failed when the object was a binary star with a period comparable or less than few times the mission length, in which case the "photocentre" had a wavy motion on the sky superimposed to the linear motion of the centre of mass. The detection in this instance was of the same nature as that of astrometric binaries on photographic plates, with the very important reservation that the Hipparcos image of an unresolved object should not be systematically identified with the photocentre.

In this paper we shall be concerned only with this second category of double stars. Our main goal is to characterize the Hipparcos astrometric binaries and to show that from the observed absolute motion on the sky it is possible to derive relevant physical parameters, like the mass ratio and the intensity ratio of the components and not simply their difference as usually done with the astrometric binaries. In fact, the possibility of investigating astrometric binaries with Hipparcos has been considered, even before launch, for example by Morel (1988) or Dommanget \& Lampens (1993), who wrote down the basic equations. The actual processing was rather different from what they had imagined, since there is no absolute observation of the motion of the primary component, but a complex signal produced by the two members of the pairs, which can be disentangled and interpreted in term of orbital motion.

To avoid the confusion between the classical astrometric binaries, based on the observation of the photocentre, and the Hipparcos astrometric binaries we coined the word hippacentre to name the physical point attached to the Hipparcos observation of an extended source. The name conveys the idea that it is very specific to Hipparcos, and that at the same time it retains some analogy with the photocentre. It is precisely defined in Sect. 2, while its induced orbital motion is investigated in Sect. 3. Finally its particular relation to the masses and luminosity of the components allows to determine simultaneously the mass ratio and the intensity ratio, provided the separation between the components is larger than about 0.3 , as explained in Sect. 4.

A companion paper in preparation will consider the applications of the principles presented here and evaluated on simulated data, to a set of about 100 astrometric binaries observed during the Hipparcos program.

\section{The astrometric information}

\subsection{Definition of the hippacentre on the Hipparcos grid}

The basic Hipparcos signal records during the transit of a star on the grid is modelled as the sum of two harmonics (Murray et al. 1989) as,

$S(t)=I+B+I M \cos (\omega t+\phi)+I N \cos (2 \omega t+\psi) \mathrm{s}^{-1}(1)$

where $I$ is the total intensity, $B$ the unmodulated background, $M$ and $N$ respectively the modulation coefficients of the first and second harmonic and $\phi$ and $\psi$ the corresponding phases. For a single star the additional relationship $2 \phi=\psi$ holds, and the value of the phase is directly linked to the direction of the star, that is to say to the photocentre of the light source.

As a result of the linearity of the Hipparcos detector, when two or more star images are simultaneously on the sensitive part of the detector their contributions add linearly and the resulting signal has exactly the same form as Eq. (1), but without the simple link between the two phases.

The positional information contained in the phase $\phi$ (indeed, we consider in the actual implementation the combination of $\phi$ and $\psi$ into a single weighted phase, but as far as the principles are concerned, this is not essential) must be related to the relative position and relative luminosity of the components. If $I_{1}, I_{2}$ are the intensities of the bright and faint component we introduce the fraction $\beta=\frac{I_{2}}{I_{1}+I_{2}}$ and the phase difference between the secondary and primary as $\xi=\phi_{2}-\phi_{1}$ (also noted $\Delta \phi$ in Mignard et al. 1995). A simple algebraic computation gives (Mignard et al. 1989, 1995),

$\phi=\operatorname{Angle}(1-\beta+\beta \cos \xi, \beta \sin \xi)=f_{1}(\xi, \beta)$

$\psi=\operatorname{Angle}(1-\beta+\beta \cos 2 \xi, \beta \sin 2 \xi)=f_{1}(2 \xi, \beta)$

where the Angle function is defined as follows:

$\operatorname{Angle}(x, y)=2 \arctan (y /(x+r))$

where $r$ is the modulus $\sqrt{x^{2}+y^{2}}$. The intensity fraction $\beta$ is related to the magnitude difference by:

$$
\beta=\frac{1}{1+10^{+0.4 \Delta m}} \quad \text { and } \quad \Delta m=-2.5 \log \left(\frac{\beta}{1-\beta}\right) .
$$

What matters now is the difference between the observed phase $\phi$ of the hippacentre and that of the primary or that of the photocentre. For the sake of simplicity consider the two stars with separation $\rho$ crossing the grid in such a way that the line between the two components is perpendicular to the slits of the grid. In this case one has $\xi=2 \pi \rho / s$ where $s$ is the gridstep of Hipparcos of size $1^{\prime \prime}$ 208. For $\rho \ll s$ one has $\xi \ll 1, \sin \xi \sim \xi$ and $\cos \xi \sim 1$, which yields with Eq. (2),

$\phi \sim \beta \xi=\beta\left(2 \pi \frac{\rho}{s}\right)$ 
which is nothing but the phase of the photocentre, since $\rho / s<1$. Thus in the limit of small separations, the hippacentre and the photocentre are alike. It is obvious from Eq. 2, that this situation does not hold true for any separation. For larger separations we have to the third order in $\rho / s$,

$\phi-\beta \xi \approx\left(-\frac{\beta}{6}+\frac{\beta^{2}}{2}-\frac{\beta^{3}}{3}\right) \xi^{3}$

which may be larger than 10 mas for $\rho>00^{\prime \prime} 3$. The difference is noticeable to Hipparcos.

Therefore, while an observation of an unresolved binary with the classical means is tied to the photocentre, the physical point (referred to as the hippacentre) attached to an Hipparcos observation of a close binary star is a point lying on the direction defined by the two components, generally between the primary and the photocentre (in some cases, the hippacentre may lie outside the segment joining the components, in the vicinity of the primary). Unlike the photocentre, the distance between the primary and the hippacentre is not a simple linear function of the separation. This will proved crucial in interpreting the path of the hippacentre on the sky induced by the orbital motion.

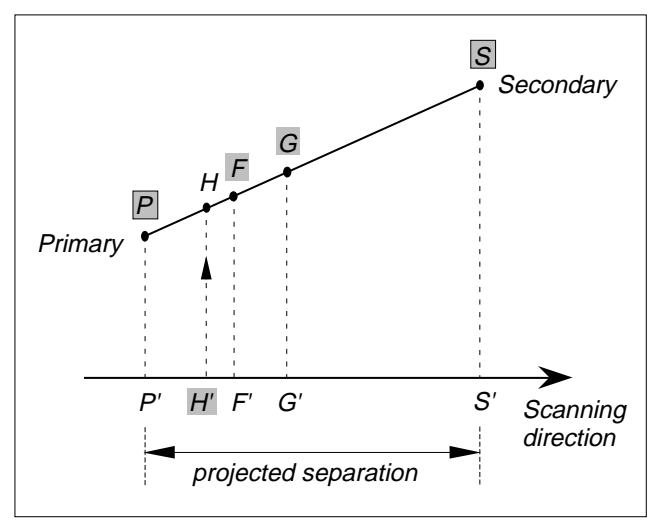

Fig. 1. Principle of the projection on the scanning circle of the points characterizing a double star. The hippacentre $H^{\prime}$ is defined on the grid but can be linked to a point $H$ on the sky. Its position with respect to the components is however variable from an observation to the other

\subsection{Absolute astrometry of a double star}

We consider now the astrometric observation of a close binary in the absence of orbital motion, in order to derive a better characterization of the hippacentre with respect to the components when the orientation with respect to the slits is arbitrary. In this case, for a given angle of scan, the positions of the primary and secondary components $P$ and $S$, the positions of the centre of mass $G$ and the photocentre $F$, are respectively projected on the scanning direction into the points $P^{\prime}, S^{\prime}, G^{\prime}$ and $F^{\prime}$, as shown in Fig. 1. The hippacentre $H^{\prime}$, initially defined on the grid, can be uniquely associated on the sky and on the line joining the two components to a point $H$, whose projection is $H^{\prime}$. In the most general case, this point is clearly different from the photocentre for $F^{\prime} \neq H^{\prime}$. More important, its relative position with respect to $P, S$ and $F$, as shown in Fig. 2, changes from one observation to another as a result of the scanning direction and the non-linearity of Eq. (2), which is not true for the photocentre.

Let $\boldsymbol{\rho}$ be the vector $\boldsymbol{P} \boldsymbol{S}$ oriented from the primary to the secondary. The positions of the centre of mass and of the photocentre are respectively given by,

$$
\boldsymbol{P G}=\frac{\mathcal{M}_{2}}{\mathcal{M}} \boldsymbol{\rho} \quad, \quad \boldsymbol{P F}=\frac{I_{2}}{I} \boldsymbol{\rho}
$$

where $\mathcal{M}=\mathcal{M}_{1}+\mathcal{M}_{2}$ is the total mass of the system. With $\beta$ and $B$ being respectively the intensity and mass fractions $\frac{I_{2}}{I}$ and $\frac{\mathcal{M}_{2}}{\mathcal{M}}$ we have for the position of the photocentre with respect to the centre of mass and its grid counterpart:

$\boldsymbol{G} \boldsymbol{F}=(\beta-B) \boldsymbol{\rho} \quad, \quad \boldsymbol{G}^{\prime} \boldsymbol{F}^{\prime}=(\beta-B) \boldsymbol{\rho}^{\prime}$

which shows that the projected distance depends on $\beta$ and $B$ only through the difference $(\beta-B)$. The corresponding equations for the hippacentre $H$ read:

$$
\boldsymbol{G}^{\prime} \boldsymbol{H}^{\prime}=\left(\frac{\phi}{\xi}-B\right) \boldsymbol{\rho}^{\prime} \quad, \quad \boldsymbol{G} \boldsymbol{H}=\left(\frac{\phi}{\xi}-B\right) \boldsymbol{\rho} .
$$

For small separations $\frac{\phi}{\xi} \approx \beta$, (Eq. 4) and there is no difference between an Hipparcos observation and the classical observation of the photocentre of an unresolved system. For larger separations the actual position of $H$ depends separately on the mass ratio and the intensity ratio and on the scanning direction, which was perfectly known in all the Hipparcos observations.

Equation (5) is related to the astrographic observations of the absolute motion of the primary of a wide pair over a long period of time. From the motion of the primary with respect to the stellar environment, one can fit by the method of least square, the two angular coordinates of the centre of mass, the two components of the proper motion, the parallax and $\frac{\mathcal{M}_{2}}{\mathcal{M}_{1}+\mathcal{M}_{2}}$.

Equation (6) is the basic relationship used in the analysis of the absolute motion of the photocenter of an unresolved astrometric binary. It shows that the photocentric orbit has the same shape as the relative orbit with a scale defined by $\beta-B$. When $\beta \rightarrow 0$, one recovers the extreme case of a binary with an unseen companion, first investigated by Bessel in the mid 19th century. In this case the photocentre and the primary coincide.

Finally, Eq. (7) relates the hippacentre to the centre of mass in more or less the same way as Eq. (6) links the photocentre to the center of mass. A fit of absolute observations carried out over a duration comparable to the orbital period will lead to the simulataneous determination 
of the astrometry of the centre of mass and the physical parameters concealed in the scale coefficient.

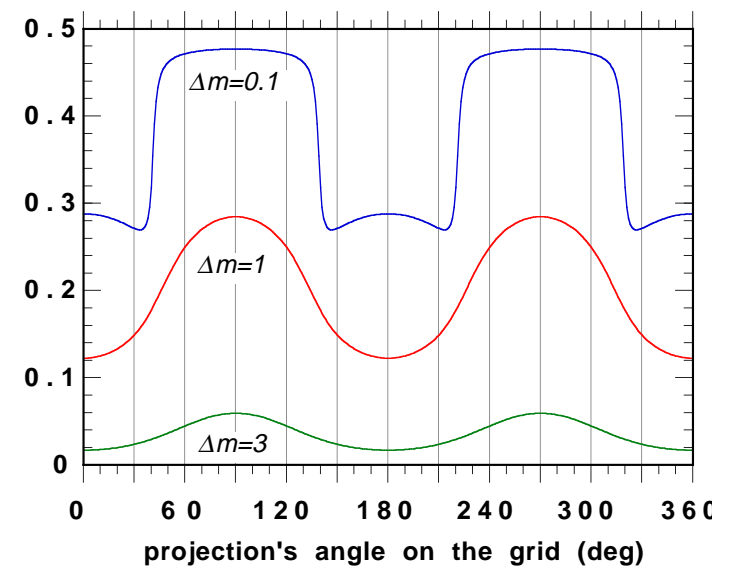

Fig. 2. Behaviour of the ratio $\frac{\phi}{\xi}$ as a function of the angle between the scanning direction and that defined by the two components, for three different values of the magnitude difference. The binary is supposed to be fix on the sky and its separation is $\rho=0$." 4 . It shows that, unlike the photocentre, the position of the hippacentre with respect to the components may be highly variable from an observation to the other

\subsection{Characterization of the Hippacentre}

Equation (7) shows that the distance between the hippacentre and the centre of mass generally includes information on the physical parameters $\beta$ and $B$ separately of one another. This very interesting feature is no longer true in three particular configurations:

- The first case arises when the separation between the components goes to zero and then $\phi / \xi \rightarrow \beta$. For a binary star with a given global magnitude $H_{\mathrm{p}}$, there is a threshold in separation below which we cannot distinguish the hippacentre from the photocentre. Taking a detection criterion of $2 \sigma$, where $\sigma$ is the standard error of the estimation of the position of the hippacentre on the grid, the limits are plotted in Fig. 3, as a function of the magnitude difference $\Delta m$. The curves are representative of the standard error for stars of magnitude 2,10, 12 and the distinction is not feasible below a particular line. From Fig. 3 one can set a broad limit of about $0 . \prime 2-0{ }^{\prime \prime} 3$ arcsec. These values will be made more precise in Sect. 5 on the basis of an extensive simulation taking account of the different steps of the processing.

- Next comes the case when the two components have similar magnitude, that is to say when $\beta \sim 0.5$. In this case, we have $\phi \approx \frac{\xi}{2}$. The ratio $\phi / \xi$ is then equal to $\frac{1}{2}$, which means that once again, the distance between the hippacentre and the photocentre is hardly discernible.

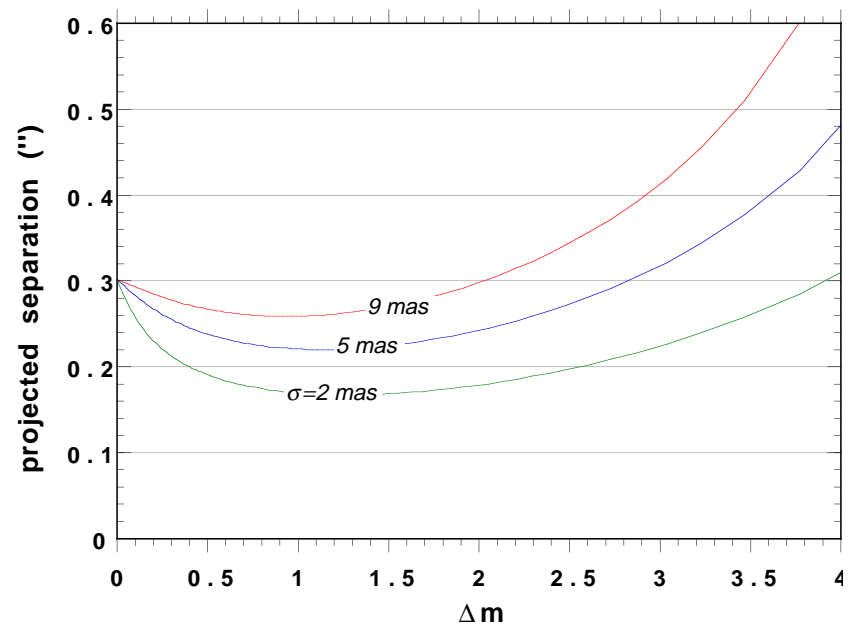

Fig. 3. Limit in separations projected on the grid, beyond which the hippacentre becomes recognizable from the photocentre, as a function of the magnitude difference and of the measurement error. The three values of $\sigma$ chosen here correspond to an Hipparcos magnitude of nearly 2, 10 and 12 mag for the system

However, with the use of the phase $\psi$ of the second harmonic, it can be shown that for projected separations larger than 0.25 gridstep (or equivalently about 0 '. 3 on the sky), the hippacentre and photocentre become clearly distinct.

- The third possibility arises just in the opposite situation when the two components are of very different brightness, corresponding to $\beta \sim 0$. The photocentre and the hippacentre are both very close to the primary and thus close to one another. In the extreme case when the companion is invisible by Hipparcos (binary with an unseen companion), the three points mix together.

In practical situations, the first limitation remains the most important, as long a it precludes any separate determination of the mass and intensity ratio.

If the semi-major axis is large enough to yield separations on the sky larger than the limit appearing in Fig. 3, when $\Delta m \approx 0$, the set of observations should contain some favourable configurations of projection on the grid, allowing the hippacentre to be sometimes distinct from the photocentre. In the third case, when the companion is unseen, the information on the value of $\beta$ is so strong $(\beta \approx 0)$ that the mass fraction $B$ may easily be derived (the phase $\phi$ is then equal to zero in any case of projection).

\section{Observation of a short-period binary}

In this section we introduce the orbital motion of the components and its observational consequences. During the 37 months of the mission, each program star has been observed at about 30 epochs, over different scanning directions. If the orbital period is large in comparison to the 
mission length, the displacement of the photocentre and of the centre of mass is just an uniform proper motion. However the hippacentre would sway in the vicinity of the photocentre with the changing scanning direction and a careful examination of the residuals could inform that the source is not a single star. Nevertheless, for most of those stars, the result would show up as an increase of the unit weight variance derived from the fit of the observations to the astrometric model.

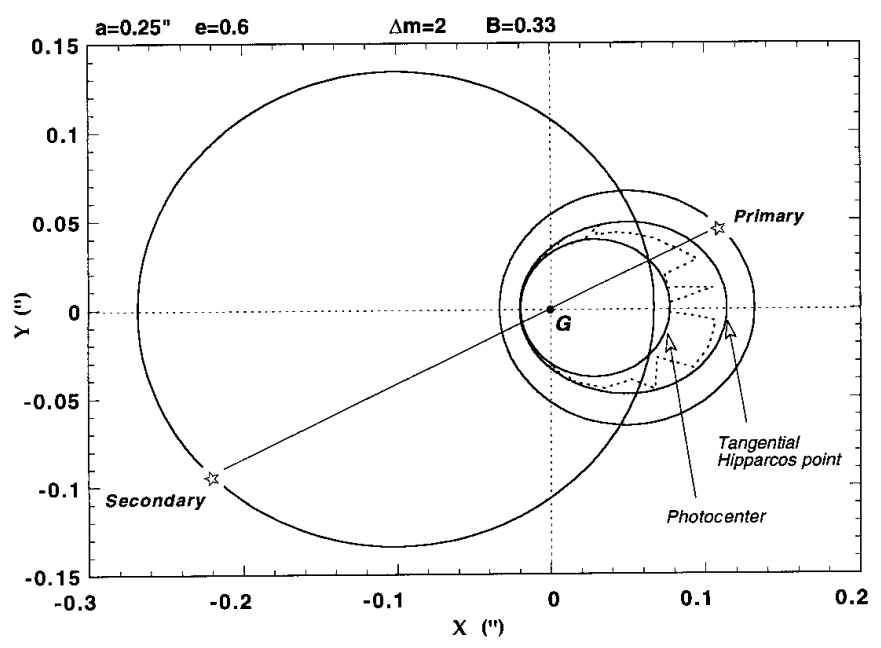

Fig. 4. Paths of the components of a double star around the centre of mass $G$, during an orbital period of nearly 3 years. $a$, $e$ and $B$ are resp. the semi-major axis, the eccentricity, and the mass fraction. About 30 observations were simulated, each one yielding to an hippacentre $H$, represented by the vertices of the dotted broken line. In this case, the position of $H$ lies between the photocentric ellipse and the line of the outer hippacentre $H^{*}$, which would occur if the projection of the double star's separation on the grid were always at its maximum. In the periastron vicinity, $H$ and $F$ are no longer distinct

\subsection{Consequences of the orbital motion}

The existence of a significant orbital motion over the duration of the mission has at least two consequences:

- The projection of the double star on the grid depends on both the scanning angle of the circle and the parameters $(\rho, \theta)$ of the binary at the time when the observations took place. Thus, the distribution of these projections is not only due to the satellite's scanning law (which depends strongly on the ecliptic latitude of the object).

- The separation $\rho$ of the pair is a function of time; the amplitude of variation depends on the eccentricity of the true orbit and on its orientation with respect to the tangential plane of the celestial sphere. As a result, even if the semi-major axis is sufficiently large, the distinction between the hippacentre $(H)$ and the photocentre $(F)$ may not be possible all along the orbit. However from the second Kepler's law, most of the orbital period is spent in the apoastron region rather than in the periastron, a circumstance which is much favourable to the detection.

In order to see the distribution of a typical set of observations of an orbital binary, we have simulated the phenomenon for various orbital parameters and with a real sequence of observations over the 37 months of the mission. The results are plotted in Figs. 4-5 for three values of the semi-major axis, assuming an orbital plane perpendicular to the line of sight. The absolute paths of the components and of the photocentre are represented. The vertices of the broken lines indicate where the hippacentres lay during these observations.

The additional curve labeled $H^{*}$ corresponds to the path that the hippacentre would have followed had the scanning direction of the satellite been always parallel to the line defined by the two components. This virtual point is noted $H^{*}$ and called outer hippacentre. One must stress that the line of $H^{*}$ is not an ellipse, and in any case never similar to the orbital paths of the components or of the photocentre. In the case of the binary star appearing in Fig. 4, the hippacentre is restricted to move between $H^{*}$ and the photocentre's and to lie between the primary and the photocentre. For separations larger than half the gridstep, this property is no longer true, as shown in Fig. $5 \mathrm{~b}$. These cases illustrate two of the many other simulations with different values of the semi-major axis $a$, the eccentricity $e$, the magnitude difference $\Delta m$ and the mass fraction $B$, carried out in order to get a better understanding of the behaviour of the hippacentre. The path of the secondary is not represented for the sake of clarity.

\section{The specific processing}

\subsection{Synthetic presentation}

We consider in this section the adaptation of the Hipparcos data processing to the case of short period astrometric binaries, in particular when the hippacentre and the photocentre are not alike. In this case, the reduction consists of producing the astrometry of the centre of mass and of determining the hippacentre path, yielding both $\beta$ and $B$. Otherwise, it yields only the scale $\beta-B$ of the photocentric orbit.

Let $S$ be a program star of the Hipparcos mission. The main goal of the standard astrometric processing was to determine accurately the position of the star with respect to the centre of mass $C$ of the solar system. When $S$ is a single star, the position vector $C S$ at epoch $t$ may be modelled with the usual five astrometric parameters:

$\boldsymbol{C S}=\boldsymbol{f}\left(l, b, \pi, \mu_{l}, \mu_{b}, t\right)$

corresponding to the coordinates of position, the parallax, and the two components of the proper motion. Although 

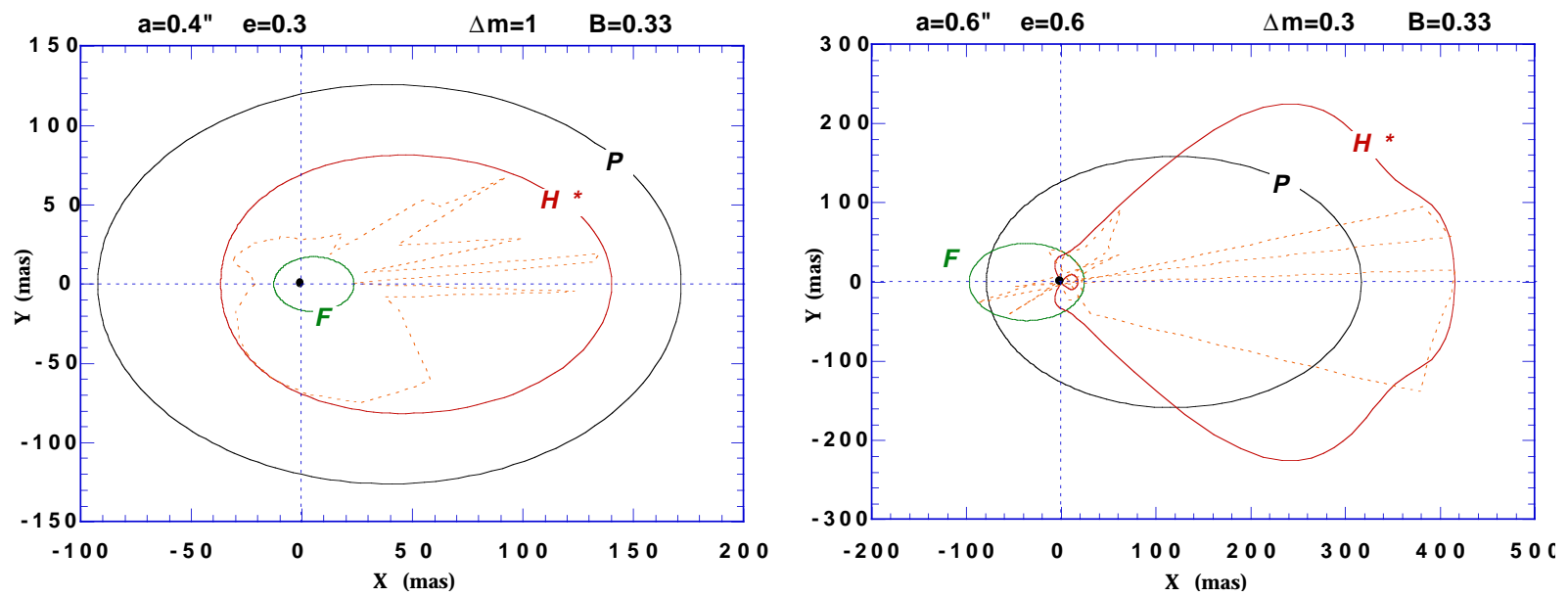

Fig. 5. a and b) Two different configurations of short-period binaries. The origin is the centre of mass. We can see in both cases the non-elliptical shape of the path of the outer hippacentre $H^{*}$. This path cannot be obtained from the photocentre by a simple dilatation

our treatment makes use of the ecliptic reference frame, this choice is of no consequence in the following.

In the case of a short period binary, the instantaneous observation is tied to the hippacentre $H$ and we have,

$\boldsymbol{C H}=\boldsymbol{C G}+\boldsymbol{G H}$

where $G$ is the centre of mass of the binary and $H$ the hippacentre, which is the same as the photocentre for very close binaries. The motion of $G$ is well described by the five astrometric parameters $l, b, \pi, \mu_{l}, \mu_{b}$, while $\boldsymbol{G H}$ is given by Eq. (7). If everything is known on the double star, $\boldsymbol{G H}$ can be computed at any time and the raw observations duly corrected for the duplicity effect and subsequently solved for the astrometric parameters of the centre of mass. If only the relative orbit is known, one must adjust in a single process both the astrometric parameters of the centre of mass and the physical parameters $\beta$ and $B$ of the binary. For separation less than $\approx 0$.' 3 , the method will fail as the hippacentre and the photocentre merge and only $\beta-B$ could be determined.

\subsection{Practical implementation}

The equations relating the observations to the astrometric and physical parameters are all non-linear. An approximate value of the position, proper motion and parallax can be used to linearize the set of equations in the vicinity of this values. No particular problem arises in this step. As for the physical parameters $\beta$ and $B$ one must be much more careful, and in most case the plain linearization is not sufficient and several tricks must be implemented to ensure the convergence during the iterations, and that the solutions we arrived at satisfies the non-linear system. With these safeguard we observed that the solution was not sensitive to the quality of the initial values of $\beta$ and $B$ and that the non-linearity problem was overcome.
The processing consisted primarily in the resolution of an over-determined system of $N$ equations (the number of observations) with five or seven unknowns, by a weighted least squares. The various steps are outlined in Fig. 6.

As the magnitude difference is generally fairly well known, the input value of $\beta$ is not totally arbitrary. Consequently, a first guess of $B$ can also be based on the mass luminosity relation for dwarf stars.

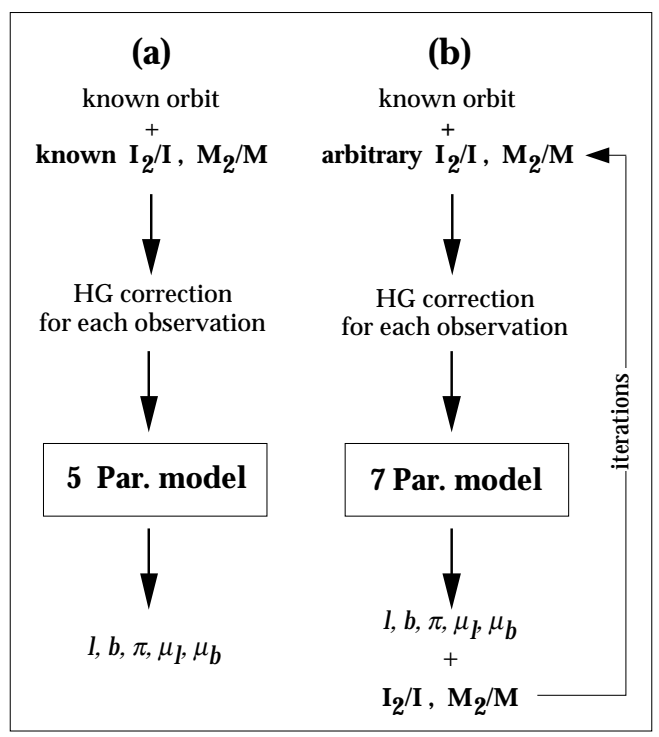

Fig. 6. Schematic principle of the specific processing of the short period binaries. In an ideal case a) where the intensity and mass fractions are perfectly known, we just use this information to shift the observations to the centre of mass and then solve for the astrometric parameters. Whenever it is not feasible b) one uses a non linear model with 7 unknowns (5 astrometric parameters for the centre of mass +2 physical terms) 


\section{The simulation}

In this section we determine the error budget of the processing and estimate the quality of the determination of $\beta$ and $B$ or $\beta-B$ according to the geometry of the double star.

\subsection{Description}

In the simulation we have generated few relatively short period binaries of various separations and computed several series of observations, using the time sequences found in the Hipparcos data sets.

According to the theoretical approach of the preceding sections, we assume that the quality of the determination of $\beta$ and $B$ or of their difference depends primarily on the three following parameters:

- the mean separation of the components on the celestial sphere over an orbital revolution,

- the orbital period $P$,

- the Hipparcos global magnitude $H_{\mathrm{p}}$.

For the sake of simplicity, each simulation refers to a particular semi-major axis $a$, which is by far the most important single geometric orbital parameter in this study. As for the other elements, each final output of the simulation is an average value (or more precisely a median) of 30 realistic cases, each resulting from a random drawing of the five remaining orbital elements, namely the inclination $i$, the eccentricity $e$, the position angles $\Omega$ and $\omega$ of the ascending node and of the periastron, and the epoch $T$. The details of this selection are really unimportant here and are not given.

The primary goal of the simulation is to determine in the space semi-major axis-period, the regions where a separate determination of the mass- and intensity-ratio is achievable and where only the scale of the photocentric orbit will be obtained. As a second objective, the simulation should also allow to analyze the effects of the choice of the initial values $\beta_{0}$ and $B_{0}$ on the convergence of the procedure, particularly in the cases of strong non-linearity (appearing when $\Delta m$ is small and the separation is about half a grid step). The third one, which is commonplace, is to help in the writing and testing of the software.

The values of the input parameters for each run, $a, P$ and $H_{\mathrm{p}}$, are given in Table 1 . This choice leads to simulate 147 basic cases $(3 \times 7 \times 7$ values), each giving rise to at least 30 random simulations as said before.

As for the other parameters they are selected as follows: the actual value of the mass fraction $B$ has no effect on the results, and thus was fixed to 0.3 in all cases, or equivalently $\mathcal{M}_{1} / \mathcal{M}_{2}=2.33$. Regarding $\beta$, apart from the very specific case with $\Delta m \approx 0$ considered in Sect. 5.3, most of the tests were run with $\Delta m=2$ or $\beta \approx 0.14$. Changing $\Delta m$ between 0.3 and 3 showed conclusively that the results were not very sensitive to any particular choice in this range. The number of observations, the position of
Table 1. Simulation's grid: values $v_{i}$ of the three input parameters

\begin{tabular}{lccccccc}
\hline Parameter & $v_{1}$ & $v_{2}$ & $v_{3}$ & $v_{4}$ & $v_{5}$ & $v_{6}$ & $v_{7}$ \\
\hline$H_{\mathrm{p}}$ (mag) & 2 & 10 & 12 & & & & \\
$a$ (arcsec) & $0^{\prime \prime} 05$ & $0^{\prime \prime} 1$ & $0^{\prime \prime} .2$ & $0^{\prime \prime} .3$ & $0 . .4$ & $0^{\prime \prime} 6$ & $0^{\prime \prime} 9$ \\
$P$ (years) & 1 & 3 & 5 & 7 & 10 & 15 & 25 \\
\hline
\end{tabular}

the simulated stars on the sky and the distribution of the scanning angles in orientation and time are chosen in order to respect the characteristics of the Hipparcos scanning law. The distance $G H$ from the centre of mass to the hippacentre was perturbed for each observation by a gaussian noise with a standard deviation function of the magnitude of the simulated system. The updating of $\beta$ and $B$ during the iterations depended on their observed correlation. For sufficiently large $a, \beta$ and $B$ are nearly independent and were separately updated. Otherwise, the relevant information is contained in the difference $\beta-B$ and the processing ended up only with a correction $\Delta(\beta-B)$. Hence, the updating was realized by splitting it into two unequal parts, as:

$$
\Delta \beta=(1-\kappa) \Delta(\beta-B) \quad, \quad \Delta B=-\kappa \Delta(\beta-B)
$$

where the coefficient $\kappa$ was generally taken equal to 0.8 , to allow for the fact that for real systems $\beta$ is as a rule better known than $B$. We observed that no more than two or three iterations were needed to reach the convergence. To start the solution algorithm we have taken $\beta_{0}=\beta-0.03$ and $B_{0}=B+0.07$, which gives a difference of 0.1 on $\beta-B$ between the true value and the initial value fed into the software.

\subsection{Assessment of the solution}

There are basically two indicators available to assess the solutions:

- The comparison of the parameters coming out of the solutions to their input values, analysed both for the bias and dispersion

- The satistical study of the standard deviations produced by the least squares packages and their comparison to the external errors.

We derived for each of the 147 basic cases, five different indicators, denoted $Q_{1}$ to $Q_{5}$. The first two are external indicators of quality, based on the comparison between the input and output values of the intensity and mass ratio, respectively $\beta, B$ and $\widetilde{\beta}, \widetilde{B}$ :

$Q_{1}=\operatorname{Med}(1.482|(\beta-B)-(\widetilde{\beta}-\widetilde{B})|)$ 

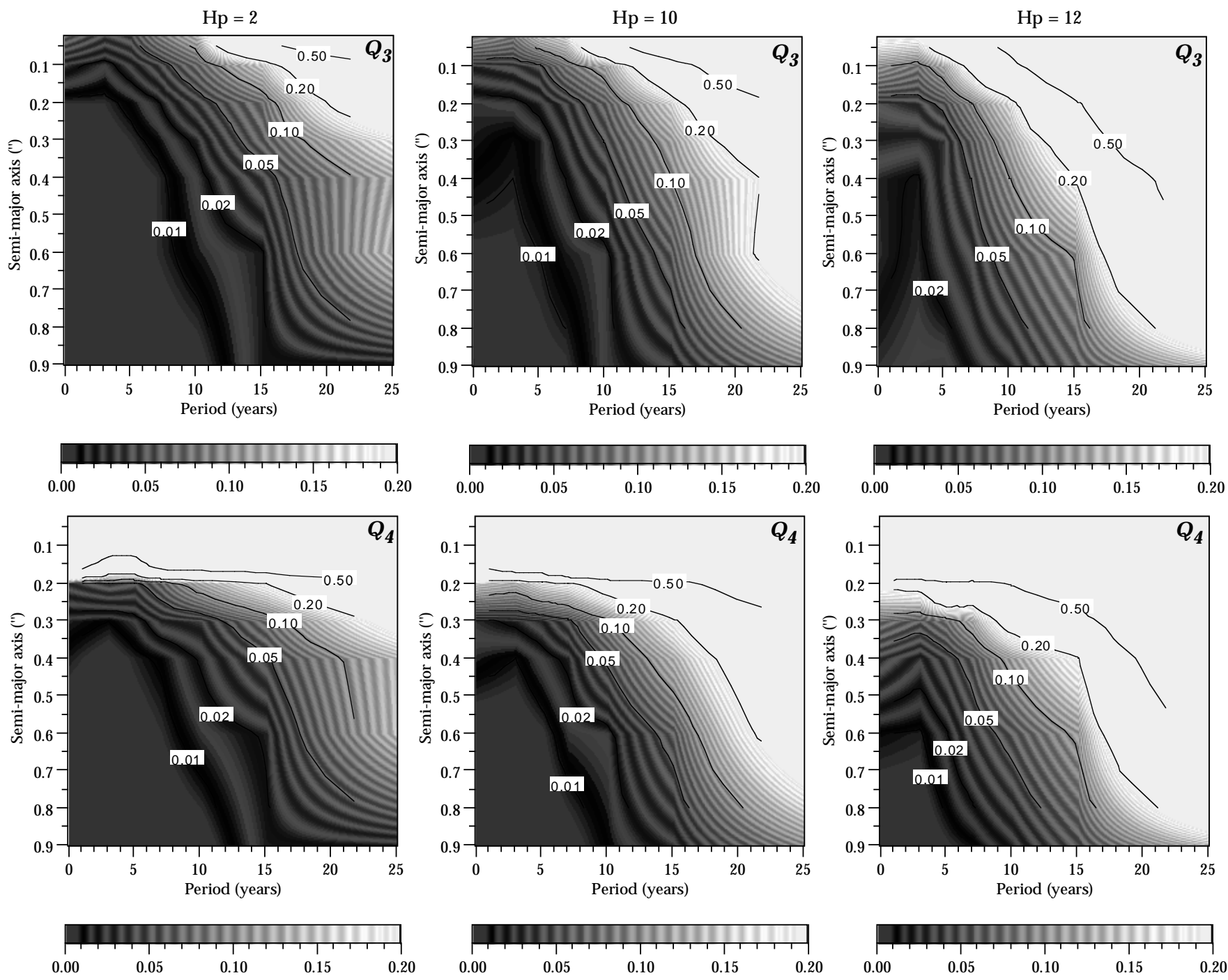

Fig. 7. Some results of the simulation $S_{1}$ (stars with $\Delta m=2$ ) for a global magnitude of 2 (left), 10 (middle) and 12 (right). The indice $Q_{3}$ (on the top) represents the standard deviation of $\beta-B$ issued from the processing, while $Q_{4}$ (on the bottom) represents the standard deviation on the mass fraction $B$ alone. Even for the shorter periods and the lowest magnitudes, it remains impossible to determine $\beta$ and $B$ separately if the semi-major axis is less than 0.2 . The constraint on the period is also crucially strengthened as the brightness decreases

$Q_{2}=\operatorname{Med}(1.482|B-\widetilde{B}|)$

where the notation Med $(\mathrm{X})$ stands for the median of the set X, with $X=\left\{x_{1}, \ldots, x_{30}\right\}$, for the 30 simulated cases (Sect. 5.1). The numerical factor in 11-12 follows from the mathematical expectation of the median of the absolute value of a gaussian random variable of zero mean and standard deviation $\sigma$, which is such that $\operatorname{Med}|X|=\sigma / 1.482$. The remaining three indices are derived from the variancecovariance matrix of the seven unknowns:

$$
\begin{aligned}
& Q_{3}=\operatorname{Med}(\sigma(\widetilde{\beta}-\widetilde{B})) \\
& Q_{4}=\operatorname{Med}(\sigma(\widetilde{B}))
\end{aligned}
$$

$Q_{5}=\operatorname{Med}(\operatorname{Cor}(\widetilde{\beta}, \widetilde{B}))$

where $\sigma(x)$ is the standard deviation of $x$, and $\operatorname{Cor}(x, y)$ is the correlation coefficient between $x$ and $y$. While $Q_{4}$ and $Q_{5}$ are directly derived from the fitting, $Q_{3}$ is evaluated from the error propagation as,

$$
Q_{3}=\sqrt{\sigma^{2}(\widetilde{\beta})+\sigma^{2}(\widetilde{B})-2 \operatorname{Cor}(\widetilde{\beta}, \widetilde{B}) \sigma(\widetilde{\beta}) \sigma(\widetilde{B})}
$$

The main results appear in Fig. 7 as maps with contour lines for the indices $Q_{3}$ and $Q_{4}$ (the indices $Q_{1}$ and $Q_{2}$ have also been plotted; As the corresponding maps are very similar to those of Fig. 7, they are not presented in this paper). The horizontal axes on each map represent the 
Table 2. List of candidate stars with possible determination of both the mass and intensity ratio

\begin{tabular}{ccccccc}
\hline HIP & HD & name & $a^{\prime \prime}$ & $P(\mathrm{yr})$ & $H_{\mathrm{p}}$ & ref. \\
\hline 171 & 224930 & 85 Peg & 0.83 & 26.3 & 5.9 & Wo \\
2762 & 3196 & 13 Cet & 0.24 & 6.9 & 5.3 & Ha1 \\
2941 & 3443 & GL 25A & 0.67 & 25.0 & 5.7 & VdB \\
7918 & 10307 & GL 67 & 0.56 & 19.5 & 5.0 & Li \\
11452 & 15285 & GL 98A & 0.54 & 25.3 & 8.8 & Wo \\
38382 & 64096 & 9 Pup & 0.57 & 23.3 & 5.3 & Ha1 \\
44248 & 76943 & 10 Uma & 0.65 & 21.8 & 4.0 & Ha2 \\
46706 & - & GL 352A & 0.55 & 18.3 & 10.0 & He \\
51986 & 92139 & p Vel & 0.34 & 16.3 & 3.9 & Wo \\
64241 & 114378 & a Com & 0.67 & 25.8 & 4.4 & Ha1 \\
80346 & - & GL 623 & 0.27 & 3.7 & 10.3 & Ma \\
84140 & 155876 & Kui 79 & 0.76 & 12.9 & 9.3 & Ha2 \\
86032 & 159561 & Rasalhague & 0.48 & 8.5 & 2.1 & Au \\
93506 & 176687 & 38 Sgr & 0.53 & 21.1 & 2.6 & Wo \\
103655 & - & GL 815A & 0.73 & 28.2 & 10.2 & Wo \\
104771 & 202128 & - & 0.23 & 5.7 & 6.3 & Ha2 \\
104858 & 202275 & 7 Equ & 0.26 & 5.7 & 4.6 & Wo \\
107522 & 206804 & GL 836.9A & 0.31 & 12.5 & 8.8 & Wo \\
\hline
\end{tabular}

Notes to Tables 2 and 3:

Au: Augensen H.J. et al. 1992; De: Demircan 0. et al. 1992; Fi: Finsen W.S. 1965; Ha1: Hartkopf W.I. et al. 1989; Ha2: Hartkopf W.I. et al. 1996; He: Heintz W.D. 1979; Hu1: Hummel C.A. et al. 1994a, Hu2: Hummel C.A. et al. 1994b; Ka: Kamper K.W. et al. 1990; Li: Lippincott S.L. et al. 1983; McA: McAlister H.A. et al. 1995; Ma: Marcy G.W. et al. 1989; Pa: Pan X. et al. 1993; VdB: Van den Bos W.H. 1937; We: West F.R. et al. 1981; Wo: Worley C.E. et al. 1983.

period in years and the semi-major axis in arcsec, while the value of each of the indicators is shown by the label of the contour lines. To fully appreciate the significance of the results shown in Fig. 7, one must first consider that the light area in the upper right of each diagram represents the domain of orbital periods and orbit size, where no valuable information on $\beta$ and/or $B$ can be extracted from the Hipparcos observations. Not surprisingly the size of this domain increases with the star magnitude. On the contrary the dark patches indicate the range of separations and orbital periods allowing the determination of the mass and intensity ratio with an acceptable accuracy. There is no hope to measure the mass ratio with the Hipparcos data for separations less than $0{ }^{\prime \prime} 15$, unless the magnitude difference is well known. In the latter case the scale of the photocentric orbit may yield a significant result even for a separation $<00^{\prime \prime} 10$.

\subsection{Special cases}

Let's come now to the limiting cases already mentioned in Sect. 2.3 when either $\Delta m \rightarrow 0$ or when the secondary
Table 3. List of candidate stars with likely determination of the scale of the photocentric orbit

\begin{tabular}{ccccccc}
\hline HIP & \multirow{2}{*}{ HD } & name & $a^{\prime \prime}$ & $P(\mathrm{yr})$ & $H_{\mathrm{p}}$ & ref. \\
\hline 12390 & 16620 & $\varepsilon$ Cet & 0.10 & 2.65 & 4.9 & $\mathrm{Ha} 1$ \\
12623 & 16739 & 12 Per & 0.06 & 0.91 & 5.0 & $\mathrm{Hu} 2$ \\
13531 & 17878 & $\tau$ Per & 0.05 & 4.15 & 4.1 & $\mathrm{De}$ \\
14328 & 18925 & $\gamma$ Per & 0.16 & 14.6 & 3.0 & $\mathrm{Hu} 2$ \\
14576 & 19356 & Algol & 0.10 & 1.86 & 2.1 & $\mathrm{~Pa}$ \\
19719 & 26690 & 46 Tau & 0.13 & 7.18 & 5.4 & $\mathrm{Ha} 2$ \\
24608 & 34029 & Capella & 0.056 & 0.28 & 0.2 & $\mathrm{Hu} 1$ \\
29850 & 43525 & 75 Ori & 0.9 & 9.20 & 5.4 & $\mathrm{Ha} 2$ \\
39261 & 65339 & 53 Cam & 0.055 & 6.64 & 6.1 & $\mathrm{Ha} 2$ \\
45170 & 79096 & 81 Cnc & 0.116 & 2.70 & 6.6 & $\mathrm{Ha} 1$ \\
45571 & 80671 & 128 Car & 0.12 & 3.2 & 5.5 & Wo \\
54204 & 96202 & $\chi 01$ Hya & 0.14 & 7.4 & 5.0 & Wo \\
75695 & 137909 & $\beta$ Crb & 0.20 & 10.5 & 3.7 & Ka \\
78401 & 143275 & $\delta$ Sco & 0.106 & 10.6 & 2.2 & Ha2 \\
83838 & 155103 & c Her & 0.11 & 8.13 & 5.5 & Ha1 \\
87895 & 163840 & - & 0.085 & 2.41 & 6.5 & McA \\
89937 & 170153 & $\chi$ Dra & 0.12 & 0.77 & 3.6 & Hu2 \\
95477 & 182369 & 47 Sgr & 0.12 & 10.8 & 5.1 & Fi \\
105431 & 203345 & - & 0.10 & 6.05 & 6.8 & We \\
112158 & 215182 & $\eta$ Peg & 0.045 & 2.24 & 3.1 & Hu2 \\
\hline
\end{tabular}

See notes below Table 2 for the references

is very faint. The tests made on systems with large $\Delta m$ confirm the conclusion of Sect. 2.3; No particular problem appears in this situation, corresponding to a classical astrometric binary with an unseen companion. The mass ratio $B$ is retrieved from the absolute motion of the primary, and this, even for separations slightly smaller than in the more common situation considered in the previous sections. On the other hand, when the two components are of similar brightness, the solving of the non linear system proved more difficult and required care in the choice of the variables. Good solutions were obtained only with periods shorter than 15 years when $a>00^{\prime \prime} 2$ and shorter than 7 years for smaller values of $a$.

\subsection{Some candidate stars}

According to the results of the simulation, it is possible to set up a list of Hipparcos stars which are likely to be good candidates to such an analysis, assuming the relative orbits are sufficiently well known. We have split this set into two categories according to whether $\beta$ and $B$ may be separately determined or not. This two sets are given respectively in Tables 2 and 3, where the values of the semi-major axis and the period are rounded. When we start the processing on real data some stars will disappear and from additional searchs in the literature we might spot new candidates. The importance of the systems belonging 
to the second category should not be underevalued; As the corresponding separations are quite small, one may find a lot of objects with extremely short periods (less than 5 years), a very favourable circumstance. One may also hope to know the magnitude difference from groundbased observations, so that the mass fraction $B$ will be also derived with a good accuracy.

\section{Conclusion}

In this paper we have identified a new category of astrometric binaries based on the peculiarities of the detection chain used by Hipparcos during the three years of its observing mission. It appears that for pairs with orbital period less than 20 years and with angular separation larger than $0 . ' 3$, it is possible to derive the mass ratio between the two components and their magnitude difference at the same time as the astrometric parameters of the centre of mass. An extensive simulation, partly based on the Hipparcos observations, has allowed to locate more accurately in the separation-period-magnitude space various regions where the processing might end up with the two physical parameters or only with their difference, as for the more common astrometric binaries. In some favorable situations an accuracy of few hundreths in the mass ratio $\mathcal{M}_{2} /\left(\mathcal{M}_{1}+\mathcal{M}_{2}\right)$ can be achieved. Finally we have identified two sets of about 20 stars each, all belonging to the Hipparcos program and having fairly reliable orbits, which will be studied soon after the release of the Hipparcos catalogue and the availability of the intermediate data.

\section{References}

Augensen H.J., et al., 1992, PASP 104, 314

Demircan O., et al., 1992, A\&A 259, 577
Dommanget J., Lampens P., 1993, Astrophys. Space Sci. 200, 221-238

Dommanget J., 1989, The Hipparcos Mission, ESA report SP 1111, Vol. II, p. 149

Dommanget J., 1985, ESA SP-234, 153-159

Finsen W.S., 1965, Rep. Obs. Circ. 7, 85

Hartkopf W.I., et al., 1989, AJ 98, 1014

Hartkopf W.I., et al., 1996, AJ 111, 370

Heintz W.D., 1979, AJ 84, 1223

Hummel C.A., et al., 1994a, AJ 107, 1859

Hummel C.A., et al., 1994b, Very High Angular Resolution Imaging, IAU, 410

Kamper K.W., et al., 1990, AJ 100, 239

Kovalevsky J., et al., 1992, A\&A 258, 7-17

Kovalevsky J., et al., 1995, A\&A 304, 34-43

Lindegren L., et al., 1992, A\&A 258, 18-30

Lippincott S.L., et al., 1983, PASP 95, 271

Marcy G.W. et al., 1989, ApJ 341, 961

McAlister H.A., et al., 1995, AJ 110, 366

Mignard F., Söderhjelm S., Bernstein H., et al., 1995, A\&A 304, 94

Mignard F., Borriello L., Kovalevsky J., Prezioso N., Bernacca P.L., 1989, The Hipparcos Mission, ESA report SP 1111, Vol. III, p. 243

Morel P., 1988, Scientific Aspects of the Input Catalogue Preparation II. In: Torra J., Turon C.(eds.) 235-244

Murray, et al., 1989, The Hipparcos mission, ESA report SP1111, Vol. III, 33-49

Pan X., et al., 1993, ApJ 413, 129

Van Den Bos W.H., 1937, Union Obs. Circ. 4, 342

West F.R., et al., 1981, BAAS 13, 569

Wielen R., 1996, A\&A (in press)

Worley C.E., Heintz W.D., 1983, The fourth catalog of orbits of visual binary stars, Publ. U.S. Naval Obs. (2) 24, part VII 